\title{
Infrared Observations of Phobos and Deimos From Viking
}

\author{
JONATHAN I. LUNINE \\ Division of Geological and Planetary Sciences, California Institute of Technology \\ Pasadena, California 91125 \\ Gerry Neugebauer \\ Division of Physics, Mathematics and Astronomy, California Institute of Technology \\ Pasadena, California 91125 \\ BRUCE M. JAKOSKY \\ Division of Geological and Planetary Sciences, California Institute of Technology \\ Pasadena, California 91125
}

\begin{abstract}
The surface thermal properties of Phobos and Deimos have been determined from observations made with the Viking Orbiter Infrared Thermal Mapper (IRTM), at wavelengths ranging from 6 to 20 $\mu \mathrm{m}$. The data, composed of both global and high-resolution infrared photometry of the satellite surfaces as well as eclipse observations, indicate surface material of low thermal conductivity comparable to that of the earth's moon. Values of the thermal inertia $/$ consistent with the data for Phobos are $0.9 \leqslant I \leqslant 1.6 \times 10^{-3} \mathrm{cal} \mathrm{cm}^{-2} \mathrm{~s}^{-1 / 2} \mathrm{~K}^{-1}$, and $0.6 \leqslant I \leqslant 2.0 \times 10^{-3} \mathrm{cal} \mathrm{cm}^{-2} \mathrm{~s}^{-1 / 2} \mathrm{~K}^{-1}$ for Deimos. It is concluded that both satellites are covered with a vertically uniform layer of finely divided material at least several centimeters thick. Observed differences between brightness temperatures at different wavelengths on Phobos are due mainly to topographic slopes and to the presence of $\sim 5 \%$ by area high inertia or blocky material.
\end{abstract}

\section{INTRODUCTION}

The infrared thermal mappers (IRTM's) on the Viking orbiters conducted extensive thermal infrared observations of the Martian satellites Phobos and Deimos at a variety of ranges, viewing geometries, and wavelengths. From these data, near-surface thermophysical properties of the satellites have been deduced. This paper describes the analysis of the IRTM data and the derived near-surface properties of Phobos and Deimos. A review of the satellites' physical properties as deduced from visual data is given by Veverka and Burns [1980].

The Martian satellites are too close to Mars as seen from earth for ground-based infrared observations to be successful. Phobos was observed by the Mariner 9 infrared radiometer, as reported by Gatley et al. [1974]. The data indicated a surface of low thermal conductivity dust at least a millimeter thick. No usable thermal observations of Deimos were made by Mariner 9 . The Viking data are of much higher quality than those obtained by Mariner 9, both because of the higher sensitivity of the Viking instrument and because most of the Viking observations were made at closer range. For those observations in which the field of view of the IRTM is entirely on the satellite, the signal-to-noise ratio achieved is up to 2 orders of magnitude greater than that of the Mariner 9 measurements.

\section{OBSERVATIONS}

IRTM instrument characteristics are detailed by Chase et al. [1978]. Briefly, the instrument is a four-telescope radiom-

Copyright 1982 by the American Geophysical Union.

Paper number 2B0970.

0148-0227/82/002B-0970\$05.00 eter operating in five infrared spectral bands centered on 7 , $9,11,15$, and $20 \mu \mathrm{m}$, with a sixth 'solar' band spanning the visible and near infrared. The $20-\mu \mathrm{m}, 11-\mu \mathrm{m}$, and solar band telescopes each have seven circular detectors arranged in a chevron pattern bore-sighted with each other and with the visual camera's (VIS) field of view (Figure 1); the fourth telescope has an identical 7-detector arrangement with 3 detectors each at 7 and $9 \mu \mathrm{m}$ and one at $15 \mu \mathrm{m}$. Each detector provides a 5.2-mrad field of view, and observations are made simultaneously in all 28 detectors. The instrument response is digitized into 1023 data numbers, which are converted into radiances based on preflight calibration and in-flight measurement of a reference plate. Zero-flux reference levels are established by pointing the instrument at space periodically. The one sample noise is approximately one data number (two data numbers for the 15- $\mu \mathrm{m}$ channel) [Kieffer et al., 1977], corresponding to temperature errors of less than $1^{\circ}$ throughout most of the range of observable temperatures when the field of view is filled; temperature uncertainties for each of the bands are given by Chase et al. [1978]. A 5\% gain drift was observed in both IRTM's during the mission, and appropriate corrections were made when spectral ratios and differences were analyzed. The bulk of the data, shown as $20-\mu \mathrm{m}$ radiances in Figures 2 and 3, consist of observations of Phobos and Deimos at phase angles up to $130^{\circ}$ and ranges greater than $1600 \mathrm{~km}$ for which the angular size of the satellite is similar to, or less than, the IRTM field of view ('whole disk' observations). Observations were also performed during eight eclipses of Phobos and four of Deimos by the shadow of Mars. In addition, eight spacecraft close approaches (range less than $1600 \mathrm{~km}$ ) of Phobos and one of Deimos occurred in which usable IRTM data were collected.

Calibration of the data was complicated by instrument 
VIKING ORBITER ।

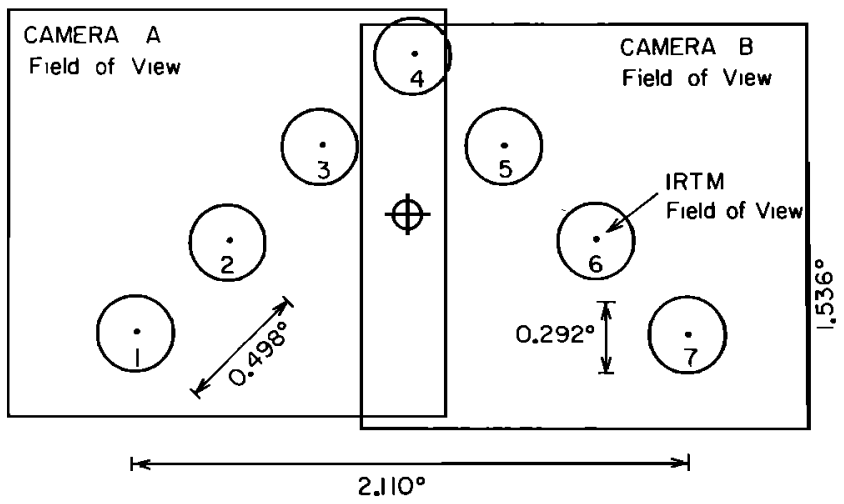

Fig. 1. Fields of view of IRTM telescopes and VIS cameras on Viking Orbiter 1. Configuration for each telescope is nearly identical, only one is shown here. Viking Orbiter 2 array is essentially the same.

pointing uncertainties and lack of simultaneous visual images for many of the observations. Because the radiometer was calibrated absolutely for sources which filled the field of view, determination of the absolute radiance depended on knowing the position of the satellite within the radiometer field of view. Predicted pointing information could not be used for this purpose since the errors in predicted satellite location were often greater than the IRTM field of view. Thus, the whole disk data in Figures 2 through 5 are to be regarded as lower bounds to the actual radiances. The large scatter in the data is due to observations in which a portion of the satellite was not in the field of view. The resolved (close approach) data were accompanied by simultaneous VIS images and hence are not subject to such uncertainty.

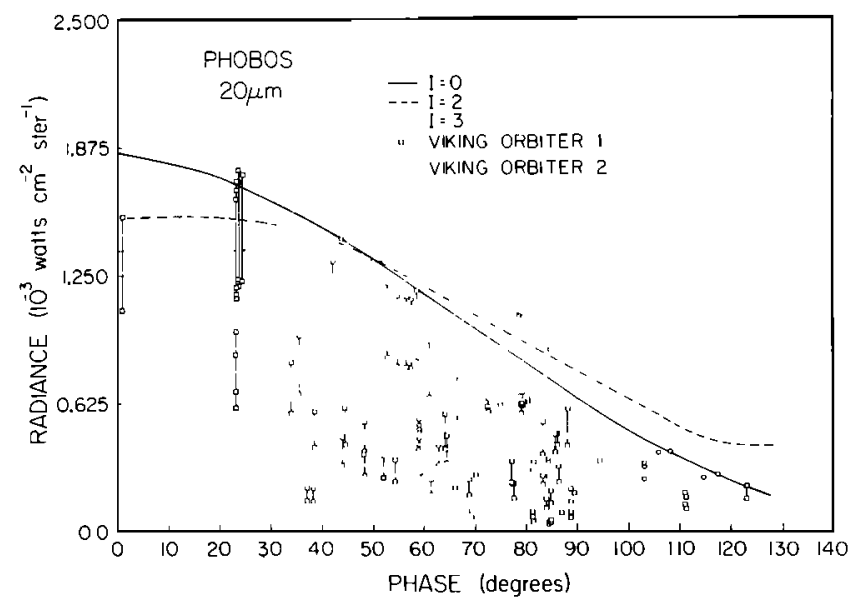

Fig. 2. Radiance versus phase angle for observations of Phobos at $20 \mu \mathrm{m}$, compared with model calculations for three values of the

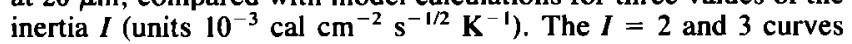
assume an averaged geometry, as indicated in text. Error bars on data points reflect uncertainties in the orientation of the satellite's major axis relative to the IRTM's line of sight. Points without error bars are observations for which projected satellite area is larger than the field of view. Instrument pointing uncertainties dictate that only the upper envelope of the data be compared with the theoretical curves.

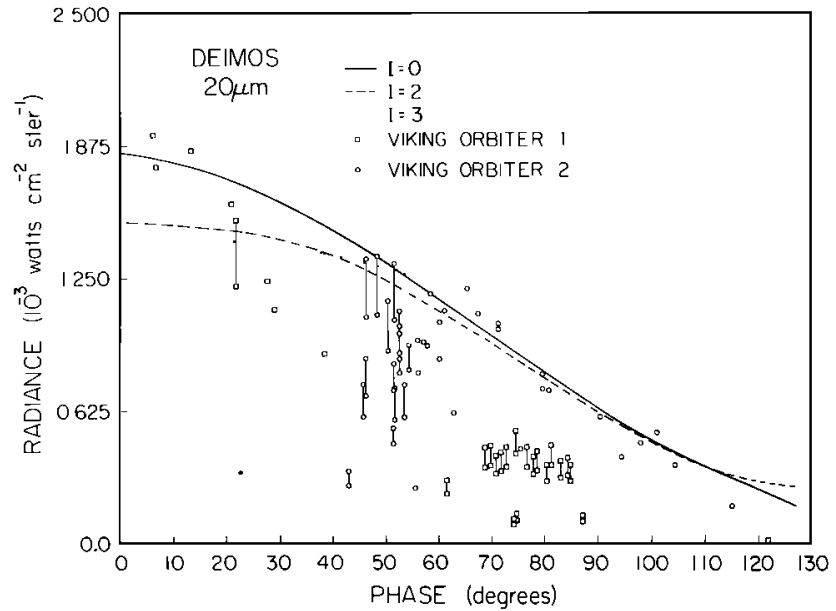

Fig. 3. The $20-\mu \mathrm{m}$ observations of Deimos (otherwise same as Figure 2).

\section{THERMAL INERTIA ANALYSIS}

\section{Whole Disk Observations}

Phase angle coverage. The whole disk observations presented in Figures 2 and 3 were compared with results from a thermal model developed by $\mathrm{H}$. Kieffer (detailed by Kieffer et al. [1977, appendix 1]). The model assumes a spherical, airless satellite and solves the heat diffusion equation for given latitude and time of day intervals with boundary conditions of no heat flow at depth and conservation of energy (in the form of incident sunlight, heat conducted inward, and surface thermal emission) at the surface [Kieffer et al., 1977, appendix 1]. The thermal characteristics needed to define the model are the emissivity $\epsilon$ and the thermal inertia $I=(k \rho c)^{1 / 2}$, where $k$ is the thermal conductivity, $\rho$ the density, and $c$ the specific heat of the nearsurface layer of the satellite. $I$ is the property which controls the temperature variation of a periodically heated homogeneous surface and hence can be employed to deduce physical properties of the observed surface [Kieffer et al., 1977]. It is given in units of $10^{-3} \mathrm{cal} \mathrm{cm}^{-2} \mathrm{~s}^{-1 / 2} \mathrm{~K}^{-1}$ throughout this paper. Except where noted below, the emissivity has been

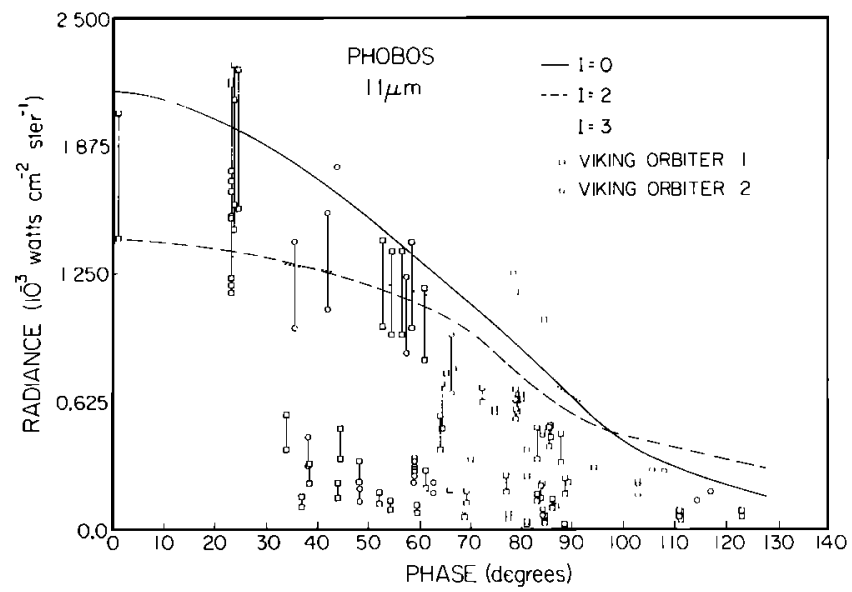

Fig. 4. The 11- $\mu \mathrm{m}$ observations of Phobos (otherwise same as Figure 2). 


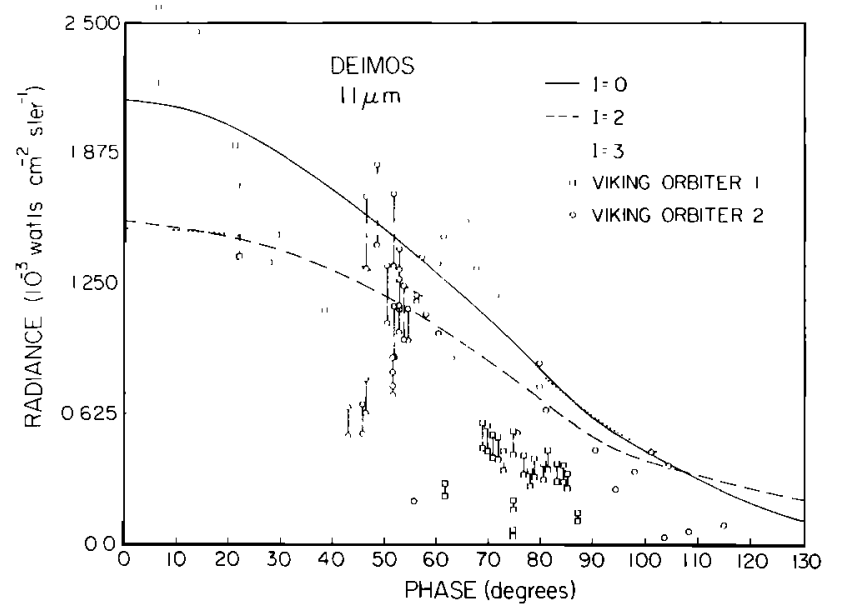

Fig. 5. The 11- $\mu \mathrm{m}$ observations of Deimos (otherwise same as Figure 2).

set equal to 1 . The bolometric bond albedo $A$ (ratio of solar energy reflected to that incident on the surface) has been set equal to 0.02 , consistent with photometric studies of Phobos and Deimos such as those by Klaasen et al. [1979]. The maximum random error in the total flux arising from the assumption that the satellites are spherical rather than ellipsoidal is $\sim 12 \%$.

Plotted on Figures 2 and 3 with the data are calculated 20$\mu \mathrm{m}$ radiometric phase curves based on the model described above for perfectly insulating $(I=0)$ and nonzero inertia bodies. The curves give brightness integrated over the whole disk of the satellite versus observed phase angle. The $I=2$ and 3 curves depend upon subspacecraft latitude and hour angle on the satellite as well as upon the phase angle; the geometry used here is an averaged one in which the subspacecraft point moves toward higher latitudes as the phase

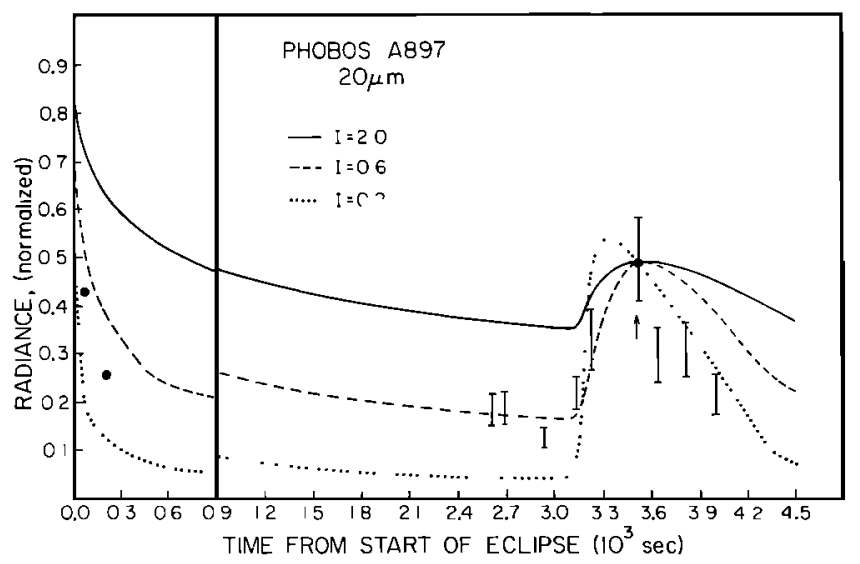

Fig. 6. Eclipse entry and exit of Phobos, plotted as 20- $\mu \mathrm{m}$ radiance versus time from start of eclipse. Model curves for eclipse exit are normalized to the data point indicated by arrow; eclipse entry curves are normalized to a preeclipse data point. Error bars carry same meaning as in Figures 2-5. Calculated sun-spacecraftsatellite geometry yields predicted orientation of satellite major axis relative to IRTM field of view at eclipse exit; this is indicated by a black dot on the exit data point. Eclipse entry curves are calculated for geometry different from exit. Since range at entry was close enough that satellite filled the field of view, major-axis orientation error bars are not shown.

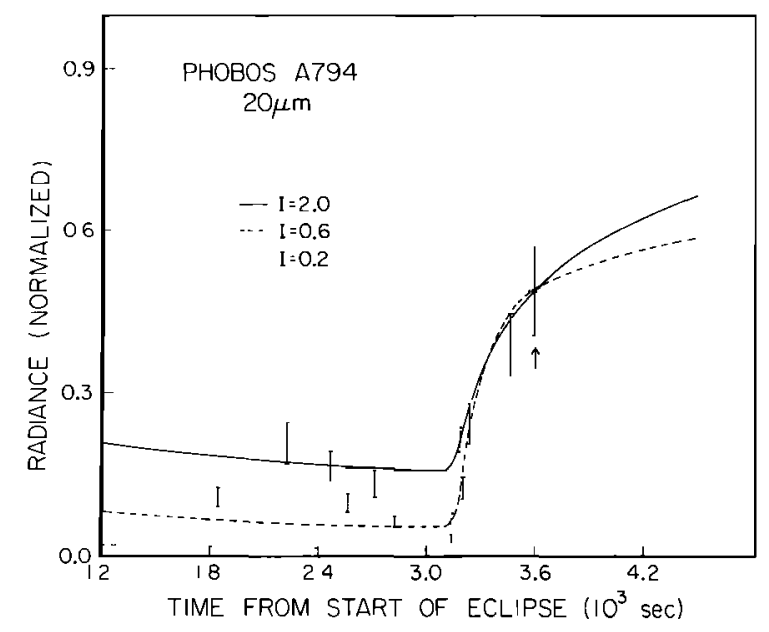

Fig. 7. Eclipse exit of Phobos at $20 \mu \mathrm{m}$ (otherwise same as Figure 6).

angle is increased. The qualitative fit to the data is unaltered if the geometry is changed. In comparing the curves with the data it must be remembered that because only a portion of the satellite may be within the field of view and the observations are not supported by VIS data, the data represent lower bounds to the actual radiances.

Given the above considerations, the phase curve formed by the upper envelope of the data is consistent with both the $I=0$ and $I=2$ curves but perhaps not with $I=3$. The fit to the $I=2$ curve is somewhat poorer than to the $I=0$ curve for phase angles greater than $90^{\circ}$ in the case of Phobos and less than $20^{\circ}$ for Deimos, suggesting that both satellites have surfaces with $I \leqslant 2$. This conclusion is consistent with that reached by Gatley et al. [1974] based on analysis of Mariner 9 radiometric data.

The 11- $\mu \mathrm{m}$ data, shown in Figures 4 and 5 , yield similar results. Notice, however, that the calculated curves fall below the upper bound of the data to a greater extent in the $11-\mu \mathrm{m}$ case than in the $20-\mu \mathrm{m}$ case, suggesting possible emissivity variation with wavelength. This will be discussed further below.

Large-scale surface irregularities may explain in part why some low phase angle observed radiances are larger than

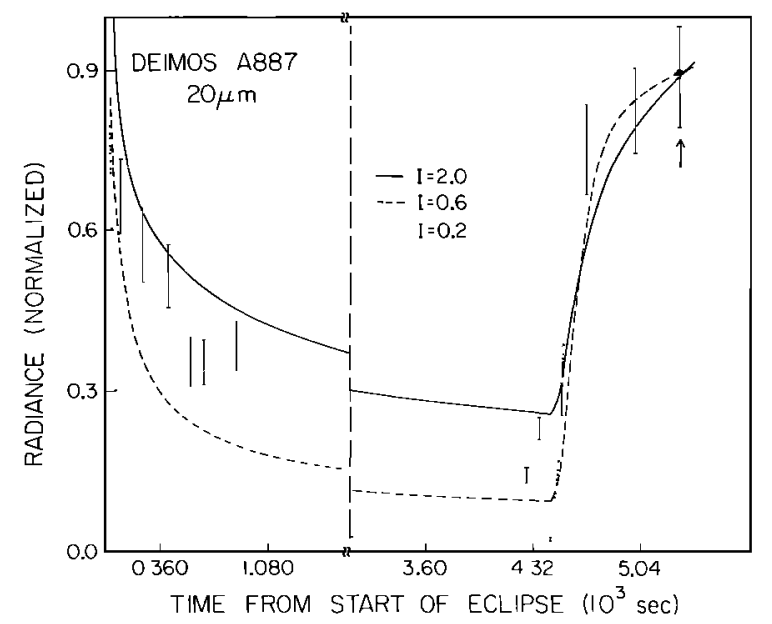

Fig. 8. Eclipse of Deimos at $20 \mu \mathrm{m}$ (otherwise same as Figure 6). 


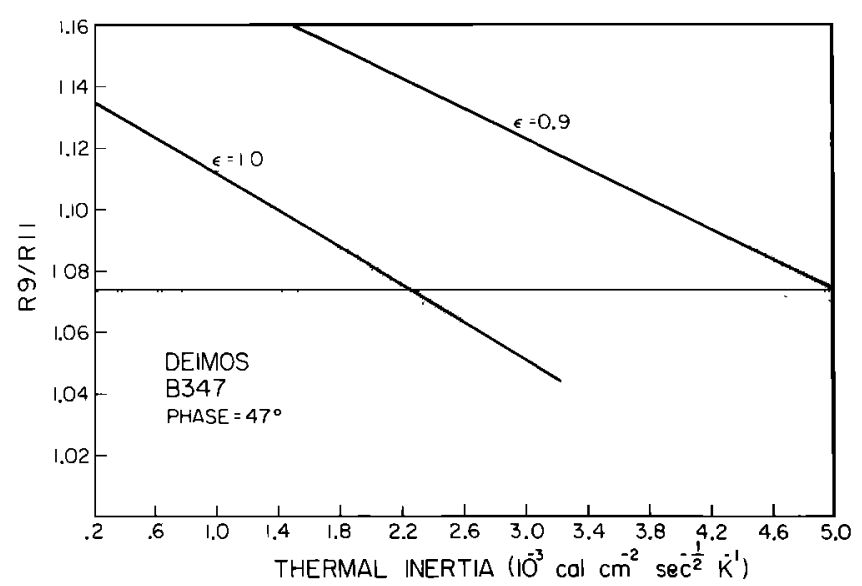

Fig. 9. Ratio of 9- to $11-\mu \mathrm{m}$ radiance $\left(R_{g} / R_{11}\right)$ for an observation of Deimos, represented as a horizontal line on the figure. Error shading represents instrument noise level. Model curves representing $R_{9} / R_{11}$ versus thermal inertia are drawn for emissivities of 0.9 and 1.0 .

those predicted by the model. At low phase angle, undulations will cause those parts of the visible surface near the limb to preferentially have lower solar incidence angles than would the surface of a sphere at the same location. Hence a larger fraction of the visible surface will experience local noon, resulting in a higher whole-disk temperature. This effect can increase the whole-disk temperature by $5-10 \mathrm{~K}$, corresponding to a 5-10\% increase in the observed radiance. At larger phase angles the effect is diminished because the temperatures near the limb are not so dependent on local time of day. At $180^{\circ}$ phase angle, the effect should be negligible.

Eclipse observations. Of the 12 satellite eclipses observed by Viking Orbiter 1, two of Phobos and one of Deimos were of sufficient overall quality to merit analysis. All three eclipses were whole-disk observations, with the angular size of the satellite comparable to, or smaller than, the field of view. The $20-\mu \mathrm{m}$ radiance data from these events are shown in Figures 6-8, plotted versus time from start of eclipse. Again, pointing uncertainties dictate that the data be regarded as lower bounds to the actual radiances. In some cases it was possible to determine the track of the satellite across the IRTM chevron pattern and identify observations for which a substantial fraction of the satellite was outside the field of view; these points are omitted from the figures. The data are compared with eclipse cooling curves calculated for $I=0.2,0.6$, and 2.0. The shape of the calculated curves is somewhat dependent on the orientation of the spacecraft relative to the sun and satellite, which was determined using satellite orbital positions computed by $\mathrm{T}$. Duxbury (personal communication, 1981). The apparently anomalous posteclipse cooling seen in Figure 6 occurs because the spacecraft was positioned beyond the evening terminator of Phobos and diurnal cooling was observed as well as the posteclipse temperature rise on the western limb. Breaks in the curve slopes in Figures 6 and 8 correspond to different eclipse entrance and exit geometries.

In the case of Phobos, data points in Figure 6 are fit most closely by the $I=0.6$ curve and certainly are consistent with curves in the range $0.6 \leqslant I \leqslant 2.0$. The data are not consistent with the $I=0.2$ curve. The mismatch between the poste- clipse cooling data and curves is most likely due to uncertainties in the precise location of the terminator on Phobos relative to the spacecraft, which significantly affects the slope of this portion of the curves. Phobos eclipse exit data in Figure 7 also generally fall within the $I=0.6$ and $I=2$ curves. The Deimos eclipse results (Figure 8) are similar; the data are best represented by curves in the range $0.6 \leqslant I \leqslant$ 2.0. Fits to the $11-\mu \mathrm{m}$ data yield identical results.

The eclipse data in Figures 6-8 can be adequately fitted by a simple 'single-layer' thermal model in which the surface has constant thermal inertia down to the depth affecting the eclipse temperatures. The scatter in the data is too large, however, to allow discrimination between the simple model and a two-layer model [see e.g., Hansen, 1973] in which, for example, a thin low inertia layer is underlain by a high inertia material. We appeal to simplicity, therefore, in concluding that the single-layer model is appropriate. Analysis of Mariner 9 eclipse data by Gatley et al. [1974] indicated a surface thermal inertia for Phobos of $\leqslant 0.5$. Considering the uncertainties in both the Viking and Mariner 9 measurements, we believe the eclipse results to be consistent with each other.

Spectral differences. An attempt was made to derive a thermal inertia for Deimos by comparing radiances from differe ${ }^{+}$spectral bands; the result is shown in Figure 9. The technique is based on the fact that a whole-disk measurement of a satellite integrates flux from many latitudes and times of day; observations at different wavelengths thus yield different radiances due to the nonlinearity of the Planck function with wavelength and temperature. The ratio of these radiances is dependent on viewing geometry, thermal inertia, and emissivity of the surface. Because emissivity variation with wavelength also changes the radiance ratios, it was necessary to select wavelength bands for which this effect was minimized. As explained below, it appears that relative emissivity effects are minimal between wavelengths of 9 and $11 \mu \mathrm{m}$; thus these channels were employed in the analysis. Emissivity variations between 20 and $11 \mu \mathrm{m}$ will be discussed in more detail below.

Because the dependence on thermal inertia of the 9- to 11$\mu \mathrm{m}$ radiance ratio is quite strong, it was necessary in the case of the whole-disk observations to select observations for which the satellite filled a significant fraction of, and was centered in, the detector field of view without overfilling it. This ensured a high signal-to-noise ratio, minimal errors in the radiances, and accurate modeling of the viewing geometry. Only observations with accompanying VIS images could be verified in this manner. One observation of Deimos by Viking 2 satisfied the criteria; no observations of Phobos were satisfactory for this analysis.

The thermal inertia values derived in Figure 9, plotted for emissivities (assumed constant with wavelength) of 1.0 and 0.9 , are no lower than 1.8. Based on the radiometric phase curve, any inertias much greater than $\sim 2.0$ are ruled out. The inertias derived in Figure 9 are suspect because of the high sensitivity of the 9- to $11-\mu \mathrm{m}$ brightness ratio to factors such as emissivity variations, geometry errors, and calibration errors. Removing a small $(\leqslant 5 \%)$ gain correction to the data, for example, has the effect of decreasing the derived inertia by $20 \%$. Hence we believe that the spectral ratio plot is not useful for fixing precise inertia values. However, the analysis does suggest that surface emissivities much less than 1.0, which yield inertias much higher than found in the phase curve data, can be ruled out for Deimos. 


\section{Spatially Resolved Observations}

The resolved data are of greater value for determining the thermal inertia because (1) the signal-to-noise ratio is quite high for a filled field of view, and (2) simultaneous VIS pictures provide accurate position information. For the Phobos data the latitude and longitude of features on the VIS pictures could be identified to within $\pm 20^{\circ}$ using coordinate grids prepared by $\mathrm{T}$. Duxbury (personal communication, 1981). It is thus possible to derive the thermal inertia from individual temperature measurements of regions on the satellite. No comparable coordinate grids have been prepared for Deimos. The surface temperature is most sensitive to inertia, and least sensitive to other effects such as surface slopes or albedo, just before dawn [Kieffer et al., 1977]. Several predawn regions on Phobos were viewed by the IRTM; none were viewed on Deimos. Figure 10 shows the result of several predawn temperature measurements; both 11- and $20-\mu \mathrm{m}$ brightness temperatures are given on the figure. The 7- and 9- $\mu \mathrm{m}$ channels have large uncertainties at the low predawn temperatures and hence were not analyzed. The wavelength dependence of the brightness temperature observed is likely to be due to the presence of different temperature materials on the surface and/or emissivity variations; these effects will be discussed further below. The observed temperatures were compared with temperatures calculated for various thermal inertias at the appropriate latitude and time of day using the thermal models described above. The rev 249 observation, made at $55^{\circ} \mathrm{N}$, is subject to large model temperature uncertainties governed by the latitude uncertainties of $\pm 20^{\circ}$ and hence is not useful for constraining the inertia. The rev 246 observations yield $0.9 \leqslant$ $I \leqslant 1.6$. An emissivity of 0.9 produces a $10 \%$ decrease in the calculated value of the inertia for rev 246 . The range in $I$ for each channel is due to latitude uncertainties of the points under observation.

\section{Effective Particle Sizes}

The above results indicate that the surfaces of Phobos and Deimos are covered with material of low thermal inertia, 0.6 $\leqslant I \leqslant 2.0$. For Phobos the inertia can be narrowed down to a range $0.9 \leqslant I \leqslant 1.6$. These values exclude solid rock, for which $I \sim 50$ [Carslaw and Jaeger, 1959, p. 497], as the dominant component of either satellites' surface. Only finely divided powders in vacuum possess the required low inertia [Wechsler et al., 1972]. For comparison, the fine particulate material covering the earth's moon has $I \sim 1$ [Winter and Saari, 1969]. The thermal inertia of Phobos and Deimos is comparable to the lowest inertias found on Mars [see Palluconi and Kieffer, 1981]. Data from Wechsler et al. [1972] indicate that for a variety of materials at low gas pressure with particle sizes in the range $35-850 \mu \mathrm{m}$, the thermal inertia of the assemblage varies from $I=0.5$ to 2.0 . We thus expect the effective particle size on the surfaces of Phobos and Deimos to average less than a millimeter and probably to be within the approximate range $50-100 \mu \mathrm{m}$.

The thermal skin depth of the dust layer is $\delta=(I / \rho c)$ $(P / \pi)^{1 / 2}$, where $P$ is the insolation period. For the eclipse data $P \sim 1$ hour; for the radiometric phase and predawn temperature data, $P$ is the diurnal period, 8 hours for Phobos and 30 hours for Deimos. Choosing $I \approx 1$ and nominal nearsurface specific heats and densities of $0.1 \mathrm{cal} / \mathrm{g} \mathrm{K}$ [Winter and Saari, 1969] and $1 \mathrm{~g} / \mathrm{cm}^{3}$, respectively, we find that $\delta$ (diurnal) $\approx 1 \mathrm{~cm}$ for Phobos and $2 \mathrm{~cm}$ for Deimos and $\delta$

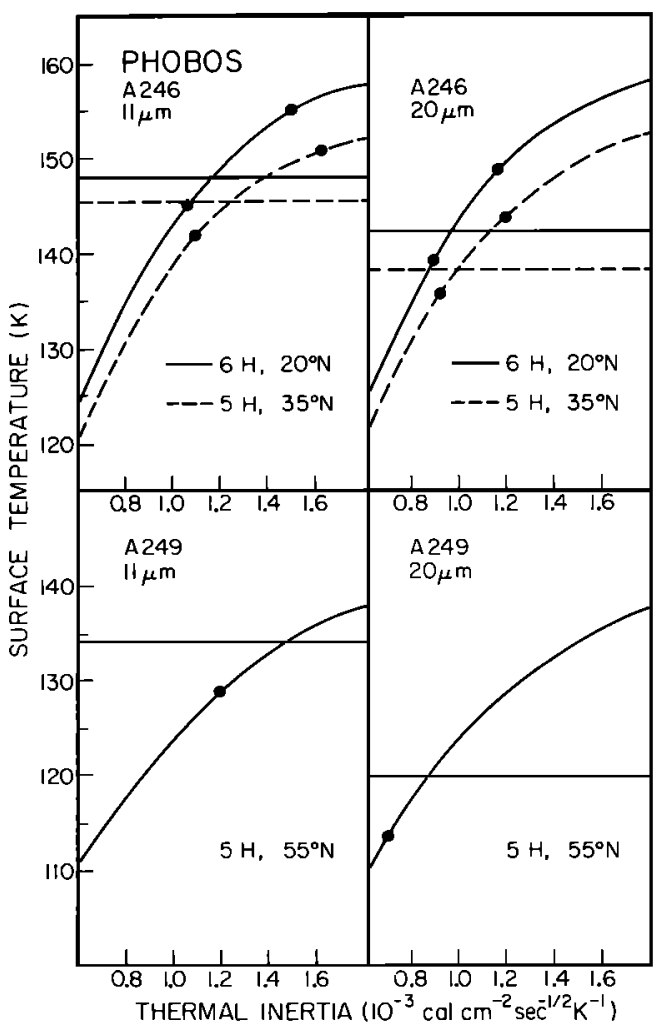

Fig. 10. Predawn surface temperatures versus thermal inertia for two sets of observations (revs A246 and A249) of Phobos at 11 and $20 \mu \mathrm{m}$. Observed temperatures are indicated by horizontal lines. Model temperature versus inertia curves are plotted corresponding to the position on Phobos of each observation; the intersection of each curve with the observed temperature yields the inertia. Uncertainties in subspacecraft latitude produce the range in the inertia values indicated by range between the dots. Upper bound inertia for A249 observation is much greater than $I=2$.

(eclipse) $\approx 0.5 \mathrm{~cm}$ for both satellites. Since both the eclipse and diurnal data can be fitted with simple single-layer models, the dust layer must be uniform over a depth range of several eclipse and thermal skin depths, i.e., $\sim 1-3 \mathrm{~cm}$ for Phobos and $1-6 \mathrm{~cm}$ for Deimos. The data are not sensitive to thermal properties below the several centimeter level, so the total depth of the dust layer is not constrained, although VIS data suggests a regolith thickness of order $100 \mathrm{~m}$ for Phobos and 10-100 $\mathrm{m}$ for Deimos [Veverka and Thomas, 1979]. Table 1 summarizes inertia values derived by the various techniques described above.

\section{Surface Spectral Properties}

In addition to the determination of averaged surface thermal inertias, the spectral characteristics of the satellite surfaces were analyzed. Since the satellite surfaces are not true blackbodies, different temperatures are derived from simultaneous measurements in each IRTM wavelength

TABLE 1. Thermal Inertia Values for Phobos and Deimos From Viking IRTM Data

\begin{tabular}{lcc}
\hline \multicolumn{1}{c}{ Technique } & Phobos & Deimos \\
\hline Phase-curve & $I \leq 2$. & $I \leq 2$. \\
Predawn temperatures & $0.9 \leq I \leq 1.6$ & $\cdots$ \\
Eclipse & $0.6 \leq I \leq 2$. & $0.6 \leq I \leq 2$. \\
\hline
\end{tabular}




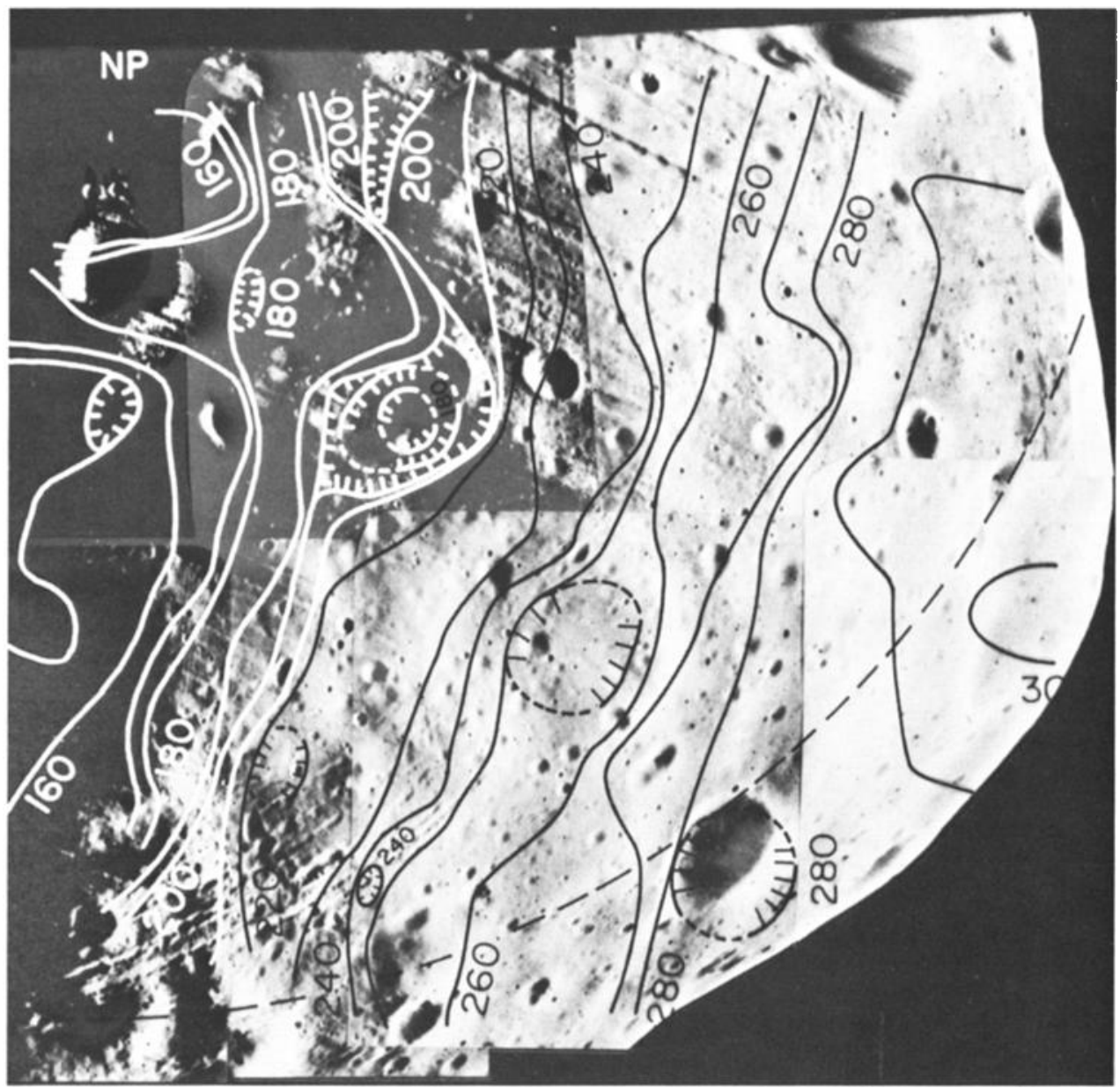

Fig. $11 a$

Fig. 11. The 11- $\mu \mathrm{m}$ (Figure 11a) and 20- $\mu \mathrm{m}$ (Figure 11b) brightness temperature contour maps of the morning hemisphere of Phobos. Contours are in $10 \mathrm{~K}$ intervals; enclosed contours of decreasing temperature are indicated by hatching. Resolution in the figure's vertical direction is somewhat poorer than IRTM field of view, while the horizontal resolution is comparable to the IRTM field of view. Dotted segments of lower temperature regions indicate contour uncertainties due to poor resolution in the figure's vertical direction. Equator and north pole are indicated in Figure $11 a$.

band. These spectral differences can, in general, arise from surface temperature variations due to topographic slopes, presence of high inertia material (or equivalently blocky material) on the surface, variation of surface albedo, nonunit emissivity, and/or emissivity variation with wavelength [Kieffer et al., 1972; Jakosky, 1979; Christensen, this issue].

Figure 11 shows 20 - and $11-\mu \mathrm{m}$ temperatures across the morning face of Phobos, overlain on a VIS mosaic of the body. Temperature deviations from the average diurnal variation are well correlated with the presence of topographic features such as ridges, crater rims, and walls. The nonblackbody character of the surface is evident in comparing the $11-$ and $20-\mu \mathrm{m}$ temperatures, with the $20-\mu \mathrm{m}$ readings being consistently lower.

It is possible, by examining the trend of brightness temperature differences as a function of time of day, to identify the major causes of the spectral contrasts on Phobos and Deimos, and hence understand the nature of their surfaces. Christensen [this issue] modeled the effects of various surface configurations on the observed spectral contrasts on Mars. He found that high-inertia materials, in the form of 10 $\mathrm{cm}$ or larger blocks, produce maximum spectral contrasts predawn and near noon, declining to near zero at 0700 and
1700 local time. Slope effects are small unless extreme, $\gtrsim 30^{\circ}$ east-west facing slopes are present, with maximum effects near 0700 and 1700 and minimum near noon and predawn. Albedo variations produce maximum contrast at 1300 hours, decreasing thereafter until dawn. Spectral contrasts due to nonunit emissivity or emissivity variation with wavelength are proportional to temperature and will therefore be lowest before dawn and peak around noon.

Diurnal spectral modeling of individual areas was not attempted because of the rough topography, which affects the temperatures as well as the lack of good diurnal coverage at any given latitude. Rather, the trend of the data was examined to isolate the major effects. The small areal coverage of the IRTM field of view means that spectral contrasts due purely to longitudinal temperature variations on a homogeneous sphere are less than $4 \mathrm{~K}$. For Phobos, $T_{11}-T_{20}(11-\mu \mathrm{m}$ brightness temperature minus $20-\mu \mathrm{m}$ brightness temperature) is maximum after dawn, decreasing toward noon (Figure 12), suggesting that steep $\left(\sim 30^{\circ}\right)$ surface slopes may be primarily responsible. The single afternoon value, which is larger than the late morning values, may indicate small-scale inertia or albedo variations, emissivity variation with wavelength, or nonunit emissivity. However, 


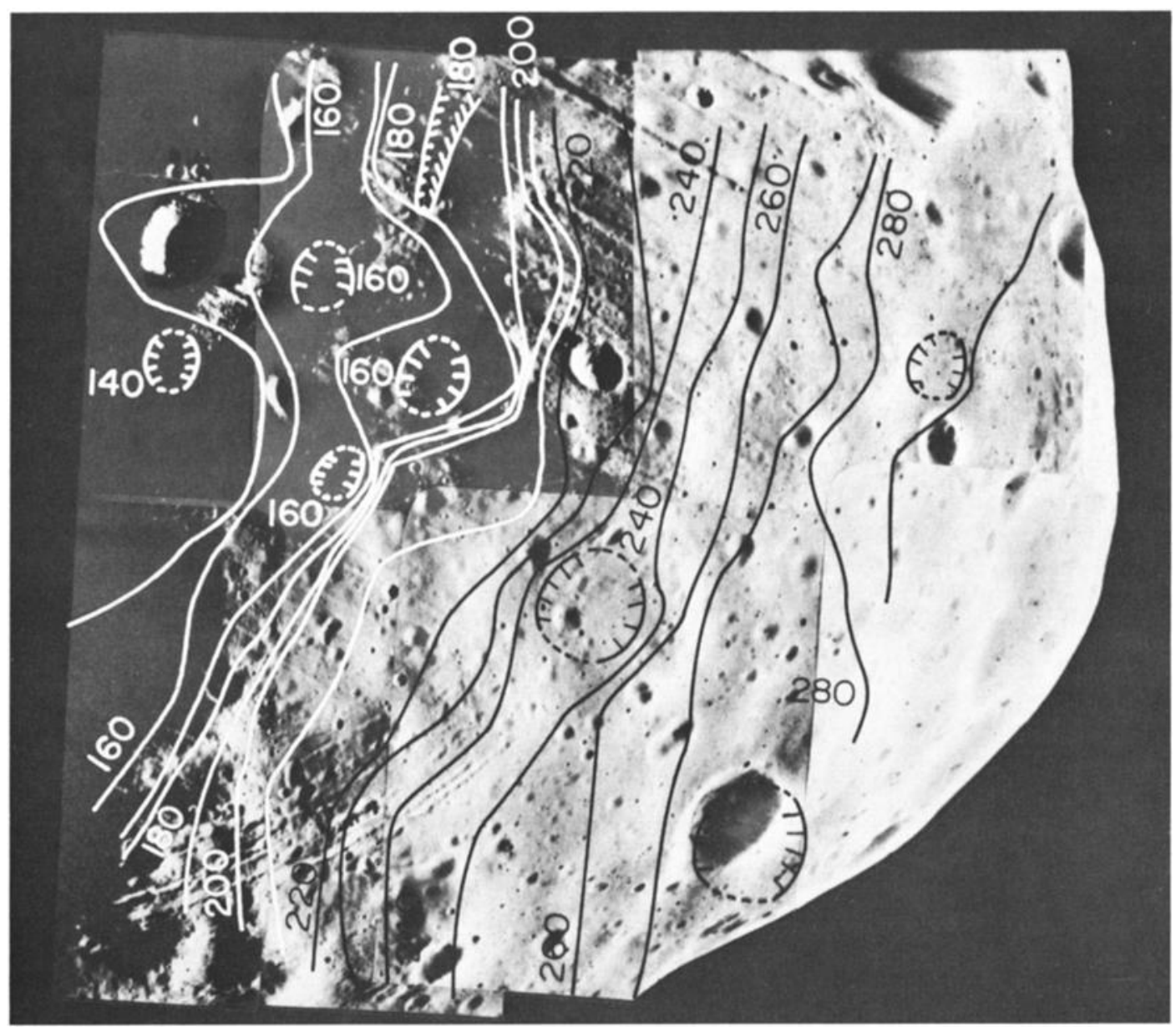

Fig. $11 b$

the viewing angle for this point was significantly more oblique than for the others, and this may also have increased the spectral contrast. The presence of a significant $(5 \mathrm{~K})$ spectral contrast before dawn suggests the presence of highinertia material, or equivalently centimeter scale blocks, on the surface. It is also possible to generate a $5 \mathrm{~K}$ spectral contrast with a ratio of 20 - to $11-\mu \mathrm{m}$ emissivities of 0.9 [Christensen, this issue]. However, the emissivity effect would then increase sharply to a noon peak of over $10 \mathrm{~K}$, according to calculations by Christensen which are generated for a surface inertia of 6.5; the lower-inertia surfaces of Phobos and Deimos would exhibit an even stronger dawn to noon $T_{11}-T_{20}$ increase due to emissivity. Figure 12 indicates late morning contrasts generally $8 \mathrm{~K}$ or less, and decreasing toward noon. Also, surface albedo inhomogeneities of $20 \%$ produce predawn spectral contrasts $<1 \mathrm{~K}$ according to Christensen's models. We thus conclude that most of the predawn spectral contrast is due to the presence of patches of high-inertia (or blocky) material. The magnitude of the maximum temperature contrast is characteristic of a surface whose primary component has $I \leqslant 2$, based on Christensen's models.

Thus on Phobos, surface slopes and patches of high-inertia material appear to be the predominant causes of spectral contrasts. Emissivity and albedo variations may be secondary causes. It is difficult to separate the emissivity effect from others producing the spectral contrasts, but based on the discussion in the previous paragraph, the data of Figure 12 place a range $1.0>\varepsilon_{20} / \varepsilon_{11}>0.9$.

Good geometry information for the IRTM observation of Deimos during the Viking 2 close approach of the satellite does not exist. However, the evening terminator direction can be identified, and the trend is toward increasing $T_{11}-T_{20}$ values as the evening terminator is approached. This suggests that surface slopes may be a predominant cause of the spectral contrasts on Deimos. Although $T_{11}-T_{20}$ is greater than $5^{\circ}$ in the early afternoon region, $T_{9}-T_{11}$ is about $1^{\circ}$ or less, suggesting that while the emissivity may be varying with wavelength, this effect is minimal for the 9- to $11-\mu \mathrm{m}$ band spectral ratio analysis shown in Figure 9. The lack of predawn and postsunset temperature data makes it difficult to constrain the presence of high-inertia material on Deimos's surface.

Using a surface blockiness model, Christensen [this issue] calculated predawn $T_{11}-T_{20}$ versus the effective thermal inertia of the composite surface for different amounts of areal coverage of $I=30$ blocks. Figure 13 shows the model results, on which are plotted Phobos predawn $T_{11}-T_{20}$ observations. The inertia values plotted for the data are based on the results of the previous section. The data indicate that the surface of Phobos is covered with approximately $5 \%$ blocky, or high-inertia, material. The result is essentially unchanged if the spectral contrast is reduced by several degrees to account for possible emissivity effects. This result is consis- 


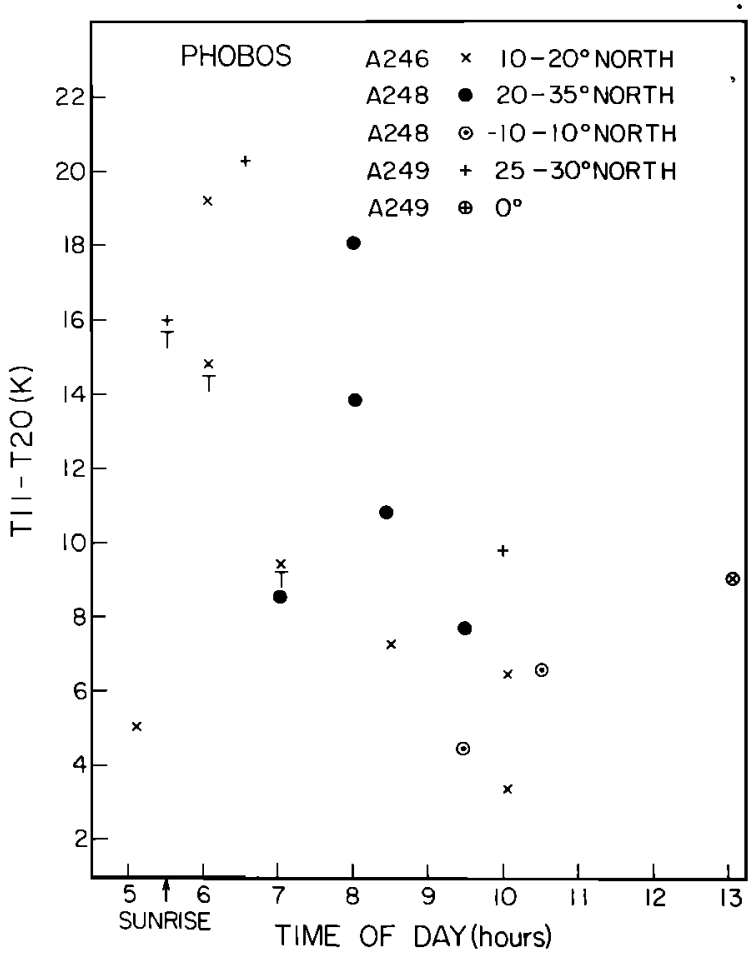

Fig. 12. $T_{11}-T_{20}$ versus time of day on Phobos. ' $T$ ' indicates points for which large-scale topographic slopes, and hence illumination and/or shadowing effects, are identifiable on VIS data.

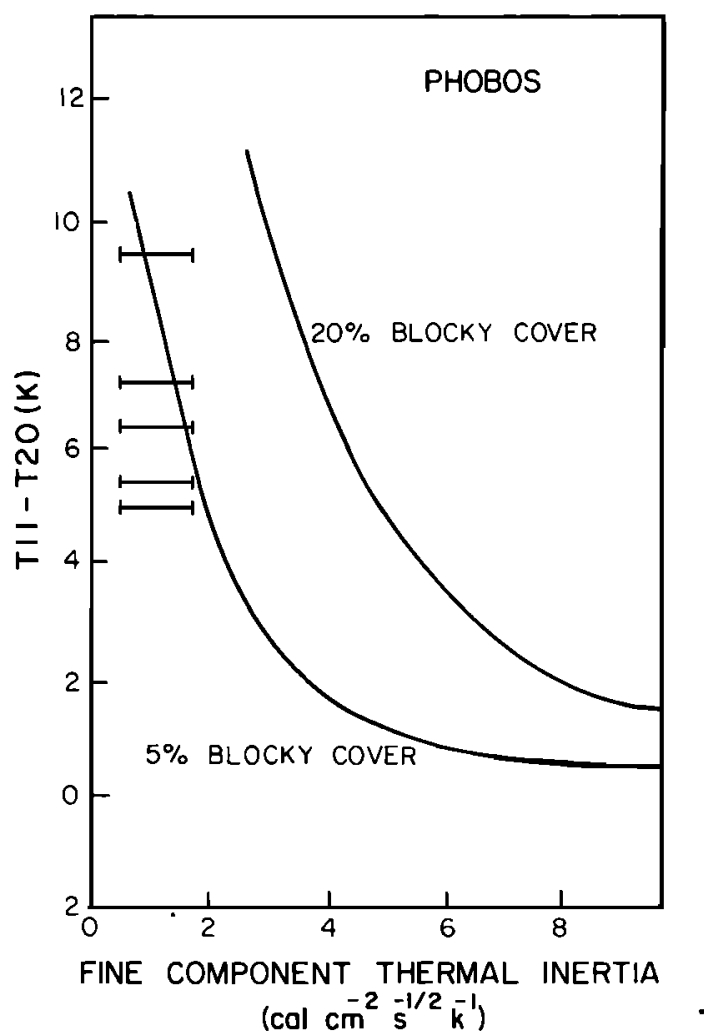

Fig. 13. $T_{11}-T_{20}$ versus effective thermal inertia on Phobos. Horizontal error bars span the thermal inertia range derived in this paper from predawn temperature data. Model curves for $5 \%$ and $20 \%$ areal coverage of surface by high-inertia $(I=30)$ blocks from Christensen [this issue]. tent with the limit of $10 \%$ set by Gatley et al. [1974], although the present data show that the areal fraction of blocky material appears to be decidedly nonzero.

\section{CONCLUSIONS}

The surface layer of Phobos is predominantly composed of low inertia $(I=1)$ material, with perhaps $5 \%$ by area blocky or high inertia material. Surface topographic slopes, possibly as steep as $30^{\circ}$, strongly influence the surface thermal response. Deimos also possesses a low-inertia surface with strong topographic slope effects; however, the extent or even existence of high-inertia material on its surface cannot be assessed. The presence of a regolith on the satellites has also been deduced from VIS data [Veverka and Thomas, 1979] and earth-based polarization studies [Veverka and Burns, 1980].

It is of interest to compare the presence of a fine regolith on the Martian satellites with what is known about asteroid surfaces. Thermal radiometry of asteroids [Morrison and Lebofsky, 1979] indicates that most objects surveyed have surfaces covered uniformly with a low conductivity regolith. Several small earth-approaching objects, including the $5-\mathrm{km}$ diameter 1580 Betulia and $2100 \mathrm{Ra}$-Shalom, do not fit lowinertia models but rather appear to possess rocky surfaces. The 433 Eros, an earth-crossing asteroid with a diameter comparable to the Martian satellites $(\sim 20 \mathrm{~km})$ which was observed with broadband spectral coverage, possesses surface thermal properties consistent with fine dust plus several percent bare rock (or an equal mix of dust and sand) [Morrison and Lebofsky, 1979].

The limited asteroid data suggest that Phobos and Deimos may be near the size limit for objects to retain a significant fine particulate surface layer. However, the environment around Phobos and Deimos is sufficiently different from that of an asteroid that the mechanisms for regolith formation and loss may be different. Recapture of escaped material may be more important for the Martian satellites than for comparibly sized asteroids, for example. Future detailed work on the nature of the asteroidal and Martian satellite regolith is needed before such problems can be addressed.

Acknowledgments. We thank Hugh Kieffer, Frank Palluconi, Ellis Miner, and Dave Paige for discussions and assistance, Judy Bennett for critical aid in processing the data, Fred Wieland for help in constructing the temperature maps, Stillman Chase for providing data on IRTM extra-field sensitivity, and Tom Duxbury for providing geodetic grids for Phobos. We are especially grateful to Phil Christensen for making available his results prior to publication as well as providing the necessary gain corrections to the data. Finally, we acknowledge the entire Viking Flight Team for carrying out the complex observations expertly. Contribution 3697 from the Division of Geological and Planetary Sciences, California Institute of Technology, Pasadena, California 91125.

\section{REFERENCES}

Carslaw, H. S., and J. C. Jaeger, Conduction of Heat in Solids, 2nd ed., Oxford University Press, London, 1959.

Chase, S. C., Jr., J. L. Engel, H. W. Eyerly, H. H. Kieffer, F. D. Palluconi, and D. Schofield, Viking infrared thermal mapper, Appl. Opt., 17, 1243-1251, 1978.

Christensen, P. R., Martian dust mantling and surface composition: Interpretation of thermophysical properties, J. Geophys. Res., this issue.

Gatley, I., H. Kieffer, E. Miner, and G. Neugebauer, Infrared observations of Phobos and Deimos from Mariner 9, Astrophys. J., 190, 497-503, 1974. 
Hansen, O. L., Ten-micron eclipse observations of Io, Europa, and Ganymede, lcarus, 18, 237-246, 1973.

Jakosky, B. M., The effects of nonideal surfaces on the derived thermal properties of Mars, J. Geophys. Res., 84, 8252-8262, 1979.

Kieffer, H. H., G. Neugebauer, G. Munch, S. C. Chase Jr., and E. Miner, Infrared thermal mapping experiment: The Viking Mars orbiter, Icarus, 16, 47-56, 1972.

Kieffer, H. H., T. Z. Martin, A. R. Peterfreund, B. M. Jakosky, E. D. Miner, and F. D. Palluconi, Thermal and albedo mapping of Mars during the primary mission, J. Geophys. Res., 82, 42494291, 1977.

Klaasen, K. P., T. C. Duxbury, and J. Veverka, Photometry of Phobos and Deimos from Viking orbiter images, $J$. Geophys. Res., 84, 8478-8486, 1979.

Morrison, D., and L. Lebofsky, Radiometry of asteroids, in Asteroids, edited by T. Gehrels, pp. 184-205, University of Arizona Press, Tucson, 1979.
Palluconi, F. D., and H. H. Kieffer, Thermal inertia mapping of Mars from $60^{\circ} \mathrm{S}$ to $60^{\circ} \mathrm{N}$, Icarus, 45, 415-426, 1981.

Veverka, J., and J. A. Burns, The moons of Mars, Annu. Rev. Earth Planet. Sci., 8, 527-558, 1980.

Veverka, J., and P. Thomas, Phobos and Deimos: A preview of what asteroids are like? in Asteroids, edited by T. Gehrels, pp. 628-651, University of Arizona Press, Tucson, 1979.

Wechsler, A. E., P. E. Glaser, and J. A. Fountain, Thermal properties of granulated materials, in Thermal Characteristics of the Moon, edited by J. W. Lucas, pp. 215-241, MIT Press, Cambridge, Mass., 1972.

Winter, D. F., and J. M. Saari, A particulate thermophysical mode] of the lunar soil, Astrophys. J., 156, 1135-1151, 1969.

(Received November 16, 1981 ; revised April 16, 1982; accepted June 28,1982 .) 